\title{
PREVALÊNCIÁ de CÁRIE deNTÁRIA EM JOVENS de 18 ANOS
}

\author{
Patrício Câmara Filho * \\ Alui Oliveira Barbisan *
CÂMARA Fọ, Patrício \& BARBISAN, Alui Oliveira. Prevalência da cárie den- tária em jovens de 18 anos. Revista da Faculdade de Odontologia. Porto Alegre, 21-22-23-24:87-101, 1979/82.

Descritores: CÁRIE DENTÁRIA, INCIDÊNCIA

\section{RESUMO}

Foram examinados 1035 estudantes do 3 ? ano do 2 ? Grau, com idade de 18 anos, em São Luís, Capital do Estado do Maranhão - Br. Os exames foram feitos com auxílio de espelho bucal plano e explorador $n$ ? 5 , para diagnosticar a prevalência de cárie, através do índice CPOD e de seus componentes, por hemiarcadas. Constatou-se que: $\mathrm{o}$ ataque de cárie chegou a $99,50 \%$ da população estudada, dando a média de 12,37 dentes CPOD por pessoa, e alcançou 44,31 dos dentes erupcionados. Houve maior ocorrência de cárie na arcada superior e, nesta, na hemiarcada esquerda. Em média, cada pessoa estava com 4,68 dentes perdidos; 4,72 , com cárie; e 2,87 restaurados. $15,10 \%$ não tinham dentes extraí- dos e $50,10 \%$ necessitavam de pelo menos uma extração dentária. $45,50 \%$ não possuíam restauração.

\section{1 - INTRODUÇÃO}

A cárie é a enfermidade mais antiga e a mais freqüente da Odontologia. Foi ela o motivo da criação de todas as escolas que formam Cirurgiões-Dentistas. É uma enfermidade irreversível e tem todas as características de um problema de Saúde Pública (CHAVES, 5).

Define-se cárie como: "uma doenca infecto-contagiosa, que se caracteriza pela desorganização dos tecidos orgânicos. É provocada pela ação de bactérias acidogênicas (sobretudo os estreptococos) na placa dental, sobre os hidratos de carbono da dieta. Como produtos finais, resultam ácidos

*Professor Auxiliar na Faculdade de Odontologia da Universidade Federal do Maranhão.

* "Mestre em Saúde Pública, Professor Adjunto e Chefe do Departamento de Odontologia Preventiva e Social, da Faculdade de Odontologia da Universidade Federal do Rio Grande do Sul.

R. Fac. Odont. P.A. 
que vão iniciar a dissolução dos prismas do esmalte, seguindo-se a ação das bactérias proteolíticas. Estas, atuando sobre a porção não mineralizada, : orgânica, vão destruí-la“"(2).

A cárie varia com a idade, sexo, raça, grau de civilização, hábitos alimentares, etc., dos povos $(22,14$, 12).

São Luís do Maranhão, - Br. onde se desenvolveu a' pesquisa, é uma ilha marítima la $44^{\circ}$ e $18^{\prime}$ a oeste de Greenwich e 20 e 32' ao sul do Equador) e seus habitantes têm predominância de descendentes da raça negra e se alimentam, em grande quantidade, de peixe e de farinha de mandioca ou arroz, principalmente os mais carentes de recursos financeiros.

Não havendo estudos epidemiológicos de cárie, em São Luís, tornou- se necessário este trabalho.

\section{2 - OBJETIVOS}

O presente trabalho objetiva descrever a prevalência de cárie dentária, utilizando o indice CPOD e seus componentes, por hemiarcada dentária.

\section{3 - REVISÃo DA LITERATURA}

O levantamento de cárie é tão necessário em uma comunidade, quanto à tomada do pulso e a pressão de um paciente, por seu médico clínico (6).

RIESER (17) em 1951, comprovou pequena ocorrência de cárie em Mossoró ( $R N-B r$.) e grande prevalência em Russas - (Ce - Br.), posteriormente.

HILL et alii (10), em 1957, estudou 8.417 crianças de 2 a 14 anos e provou que crianças brancas de ambos os sexos, tiveram mais cárie que crianças pretas.

JACKSON (11), em 1961, estudou cárie em adultos, e no grupo de 15 a 19 anos, encontrou $37 \%$ de dentes afetados e que a cárie cresceu $2,6 \%$ em média ao ano, entre as idades de 12 a 17 anos; e 1,20\% ao ano, nas idades de 19 a 32 anos e 0,3\% ao ano, nas idades de 32 a 47 anos.

JOHNSON et alii (12), em 1962, constataram que nas idades de 18 a 79 anos, mais de 20 milhões de americanos perderam todos os seus dentes e, aproximadamente, 10 milhões perderam todos os 16 dentes de uma das arcadas. O CPOD médio foi de 20,40 assim distribuídos:

$\begin{array}{ccc}\text { cariados } & \text { perdidos } & \text { restaurados } \\ 1,20 & 13,50 & 5,70\end{array}$

Entre 14 e 18 anos, 1,3\% e 1,4\%, dos homens e das mulheres, respectivamente, eram desdentados.

CHAVES (7), em 1965, encontrou, na faixa etária de 16 a 21 anos, CPOD de 12,12, assim distribuído:

cariados perdidos restaurados

$7,20 \quad 3,70 \quad 1,22$

TINOCO (20), em 1966, estudando prevalência de cárie na Cidade de Areia Branca, comprovou haver baixa prevalência dessa enfermidade na população de 1 a 14 anos de idade. Encontrou baixo teor de fluor na água de abastecimento, sugeriu novos levantamentos de cárie, nova análise da água e pesquisas de hábitos alimentares, naquela comunidade.

SOUZA et alii (18), em 1967, encontraram CPOD de 8,58 para crian. cas brancas e 6,78 para crianças pretas, ambas com idade de 12 anos na Capital Paulista. 
O DEPARTAMENTO DE ESTA. TISTICA DOS ESTADOS UNIDOS (21), em 1967, publicou que $91 \mathrm{mi}$ Ihões de adultos tiveram CPOD de 17,80 , assim distribuído:

cariados perdidos restaurados

$$
\begin{array}{lll}
1,40 & 9,40 & 7,00
\end{array}
$$

Destaca que, as médias de $13,40 \mathrm{e}$ 14,10, para adultos jovens, masculinos e femininos, respectivamente (sem precisar a idade), foram crescendo com a idade, de modo que na faixa etária de 75 a 79 , alcançaram os 25 dentes por pessoa.

SUMMERS (19), em 1970, examinou estudantes de 12 anos e obteve valores médios: para crianças negras: 3,6 para masculino e 5,72 para sexo feminino; para crianças brancas: 3,61 para masculino e 5,89, para feminino.

MEJIA et alii (14), em 1971, em Bogotá - Colômbia - examinando 14.296.000 pessoas de 5 a mais anos, e obtiveram CPOD médio de 15,40 , com a seguinte distribuição:

$\begin{array}{cc}\text { cariados } & \text { ext. indic. } \\ 4,00 & 1,40 \\ \text { extraídos } & \text { restaurados } \\ 9,00 & 1,00\end{array}$

No grupo de 15 a 19 anos o CPOD chegou a 13,10, em média, por pessoa, o que corresponde a $49 \%$ do total de 28 dentes, que deveriam estar erupcionados.

MEDEIROS (13), em 1971, examinou 799 indivíduos do sexo masculino, em São Paulo (Capital), com idades de 18 a 50 anos. Para o grupo de 18 a 20 anos, encontrou a média de 24,04 dentes presentes, a o CPOD médio de 15,04, assim distribuído:

$\begin{array}{cc}\text { cariados } & \text { ext. indic. } \\ 6,37 & 1,21 \\ \text { extraídos } & \text { restaurados } \\ 5,96 & 1,50\end{array}$

BAGRAMIAN \& RUSSEL (1), em 1971, examinaram jovens de ambos os sexos, pretos e brancos, de 2 cidades diferentes, (Detroit, ao norte de Michigan e Columbia, ao sul de Carolina) de idades de 14 a 17 anos. Para a idade de 17 anos obteve valores que variaram de 10,23 a 12,83 , para os brancos e 10,22 a 11,84, para os pretos.

PINTO (16), em 1971, estudando população de 15 a mais anos na Vila Passo das Pedras em Porto Alegre RS - obteve, em média, os seguintes resultados:

$\begin{array}{cc}\text { cariados } & \text { ext. indic. } \\ 3,38 & 1,59 \\ \text { extraídos } & \text { rest. } \\ 8,41 & 1,43 \\ \text { CPOD } & \mathrm{C}+\mathrm{Ei} \\ 14,81 & 4,97\end{array}$

GUIMARÃES (8), em 1972, estudou risco de cárie, concluindo que as meninas são mais atacadas por cárie; que $o$ ataque em ambos os sexos cresce com a idade; afirma que o risco de cárie em dentes homólogos é estatisticamente igual ao nível de significância de 5 por cento. Diz que o risco de cárie pode ser calculado com o exame de dois hemiarcos.

CAHEN et alii (3), em 1977, encontraram CPOD de 12,60 para 0 grupo de jovens de 18 anos. Constataram que, aos 12 anos, $19,10 \%$ dos dentes haviam sido atacados pela cárie; que essa porcentagem chegou a $30,90 \%$ aos 15 anos e que alcançou $43,5 \%$ na faixa etária de 16 a 18 anos, permanecendo, ainda, por volta de $47 \%$, aos 30 anos.

CAMPANELLA \& CICIARELLI (4), em 1977, obtiveram, para crian- 
ças de 14 anos, CPOD de 11,83, distribuído assim:

$\begin{array}{cc}\text { cariados } & \text { extraídos } \\ 5,51 & 0,86 \\ \text { ext. indic. } & \text { restaurados } \\ 0,45 & 5,01\end{array}$

Observaram que $39,41 \%$ dos dentes foram afetados por cárie, nessa idade.

HANSEN (9), em 1977, encontrou, para a idade' de 35 , anos, em Oslo (Norway), CPOD de 25,70; com a média de 5,1 dentes cariados por pessoa e 18,9 restaurados, também em média, por pessoa.

MOREIRA \& VIEIRA (15), em 1977, examinaram crianças brancas $e$ pretas da cidade de Piracicaba - SP $\mathrm{Br}$.

Para a idade de 12 anos, obtiveram CPOD: para masculino: 6,83 para pretas e 7,57 para brancas; para o feminino, 7,94 para pretas e 9,43 pa. ra brancas.

Sendo a literatura escassa em estudos de cárie em idades de 18 anos separadamente, foram citados aqui os resultados das idades mais próximas de 18 anos e de faixas etárias que a englobam.

Optou-se por fazer-se este trabalho em jovens de 18 anos, visto ser a idade de início de produtividade econômica e quando os jovens de sexo masculino enfrentam a fase mais importante de suas obrigações militares.

\section{4 - MATERIAL E MÉTODOS}

\section{1 - População estudada}

Foram examinados 1035 jovens de 18 anos, que frequentavam a 3 ? série do $2{ }^{\circ}$ Grau do turno matutino, da Cidade de São Luís, Capital do Estado do Maranhão.

\section{2 - Método de coleta de dados}

Todos os exames foram feitos por um único examinador $e$ as anotações foram feitas por estudantes concludentes do Curso de Odontologia. Foi utilizada uma ficha que se destina, também ao levantamento de necessidades protéticas. $O$ exame foi executado com espelho bucal plano e explorador $n$ ? 5, com visão direta, à luz natural, nas próprias escolas.

\section{3 - Códigos e critérios que foram utilizados}

Os códigos utilizados foram os de Klein Palmer e os critérios, os que são utilizados pela Faculdade de Saúde Pública da Universidade de São Paulo.

\section{5 - RESULTADOS}

Foram perdidos, em média, 4,68 dentes por pessoa. Mais de 2/3 dos perdidos já haviam sido extraídos $(3,26)$ e apenas 1,42 em média, por pessoa, estavam com indicação de extração. Essas extrações indicadas não estavam eqüidistribuídas já que a maioria não as possuíam (moda 0,00 - Tab. 1).

A média de dentes cariados foi de 4,72, próximo da mediana $(4,51)$, com erro padrão de 0,11 (Tab. I).

Para restaurados, a média foi de 2,97 dentes por pessoa; com erro padrão 0,12 e moda zero $(0,00)$, indicando assim que a maioria dos exa. minados não possuíam dentes restaurados e eram poucos os que possuíam grande número de dentes nessa condição (Tab. I).

Em média, 6,14 dentes por pessoa precisavam de cuidados odontológicos de restaurações e de extrações (C + Ei Tab. 1). $44,31 \%$ de todos os dentes foram afetados por cárie. 
0 índice CPOD médio foi de 12,37 , com valor igual ao da mediana, com moda 11,00 e erro padrão 0,17 . Portanto, quase metade dos dentes foram atacados por cárie (Täb. I).

Para a condição de hígido, os valores da mediana $(15,58)$ e da moda $(15,00)$, foram aproximadamente imais, e a média foi de 15,55 (Tab. I).

\section{Dentes Extraídos}

De todos os examinados, apenas $15,70 \%$ não tinham dentes extraídos; $17,50 \%$ haviam extraído apenas um dente; $21,30 \%, 15,00 \%$ e $11,30 \%$ tinham, respectivamente, 2,3 e 4 extraçöes feitas; e apenas uma pessoa tinha 17 dentes nessa condição (Tab. II).

\section{Dentes com extraçáo indicada}

Quase a metade dos examinados não necessitava de extração $(49,90 \%)$; $19,80 \%$ necessitavam de extração de apenas um dente e $12,40 \%$, de 2 dentes; só um indivíduo necessitava de 21 extrações (Tab. II).

Houve mais indicação de extração na arcada superior $(5,63 \%)$ que na in. ferior $(4,55 \%)$. Na hemiarcada superior esquerda houve maior número de dentes com extração indicada (correspondendo a $5,76 \%$ dos dentes); sendo que a inferior esquerda teve menor porcentagem $(4,41 \%$ dos dentes - Tab. III e IV).

\section{Tabela I}

Medidas de tendência central e de variabilidade das condições dos dentes de 1035 jovens de 18 anos

São Luís do Maranhão 1978.

\begin{tabular}{lrrrrr}
\hline Condição & $\begin{array}{r}\text { No de } \\
\text { dentes }\end{array}$ & Média & Mediana & Moda & $\begin{array}{c}\text { Erro } \\
\text { Padrão }\end{array}$ \\
\hline Extrardo & 1.379 & 3,26 & 2,29 & 2,00 & 0,08 \\
Ext. Ind. & 1.469 & 1,42 & 0,51 & 0,00 & 0,07 \\
Perdido & 4,848 & 4,68 & 3,50 & 2,00 & 0,13 \\
Cariado & 4.888 & 4,72 & 4,51 & 2,00 & 0,11 \\
Restaurado & 3.077 & 2,97 & 1,02 & 0,00 & 0,12 \\
CPOD & 12.813 & 12,37 & 12,37 & 11,00 & 0,17 \\
Higido & 16.099 & 15,55 & 15,58 & 15,00 & 0,17 \\
\hline
\end{tabular}

Foram extraídos mais dentes na arcada inferior $(12,63 \%)$ que na superior $(10,74 \%)$. Houve maior freqüência de dentes extraídos na hemiarcada inferior direita $(13,03 \%)$, ficando a superior esquerda com a menor freqüência $(10,23 \%)$ (Tab. III e IV).

\section{Dentes Perdidos}

Houve mais perda de dentes inferiores $(17,18 \%)$, que de superiores $(16,37 \%)$. 
Em ambas as arcadas, nos dentes do lado direito, houve mais perda $(17,60 \%$, para a inferior e $16,75 \%$, para superior) que nos do lado esquerdo $(16,65 \%$, na inferior $(15,99 \%$ na superior - Tab. III e IV).

\section{Dentes Cariados}

Não tinham dentes cariados $10,00 \%$ das pessoas examinadas; $9,30 \%$ tinham apenas um dente com cárie. Com 2,3,4,5,6 e 7 dentes cariados, as porcentagens de examinados variaram de $9,30 \%$ a $10,70 \%$ para cada um desses grupos. Apenas um examinado tinha 18 dentes cariados, e outro, 21 dentes nessa condição (Tab. II).

Observa-se, na Tabela III, que a arcada superior apresentou maior porcentagem de dentes cariados $(20,60 \%$ dos dentes) e, nesta, a hemiarcada esquerda (com 23,25\%) superou a direita $(17,96 \%)$; ficando a inferior direita com a menor porcentagem (13,15\% - Tab. III).

\section{Tabela II}

Distribuição numérica e percentual de 1035 jovens de 18 anos, de ambos os sexos, por CPOD e seus componentes, segundo o $n$ ? de dentes afe tados pela cárie $\mathrm{S}$. Luís (MA)

1978

\begin{tabular}{|c|c|c|c|c|c|c|c|c|c|c|c|c|c|}
\hline \multirow[b]{2}{*}{$\begin{array}{l}\text { No de } \\
\text { Dentes }\end{array}$} & \multirow{2}{*}{$\begin{array}{c}\text { Condição } \\
\text { Pessoa }\end{array}$} & \multicolumn{2}{|c|}{ Cariado } & \multicolumn{2}{|c|}{ Extraído } & \multicolumn{2}{|c|}{$\begin{array}{l}\text { Extração } \\
\text { Indicada }\end{array}$} & \multicolumn{2}{|c|}{ Restaurado } & \multicolumn{2}{|c|}{ CPO } & \multicolumn{2}{|c|}{ Hígido } \\
\hline & & № & $\%$ & № & $\%$ & No & $\%$ & № & $\%$ & No & $\%$ & N? & $\%$ \\
\hline 0 & & 103 & 10,00 & 163 & 15,70 & 516 & 49,90 & 471 & 46,50 & 5 & 0,50 & 4 & 0,40 \\
\hline 1 & & 96 & 9,30 & 181 & 17,50 & 205 & 19,80 & 89 & 8,60 & 7 & 0,70 & 3 & 0,30 \\
\hline 2 & & 111 & 10,70 & 220 & 21,30 & 128 & 12,40 & 68 & 6,60 & 13 & 1,30 & 5 & 0,50 \\
\hline 3 & & 99 & 9,60 & 155 & 15,00 & 64 & 6,20 & 65 & 5,30 & 24 & 2,30 & 5 & 0,50 \\
\hline 4 & & 108 & 10,40 & 117 & 11,30 & 33 & 3,20 & 45 & 4,30 & 26 & 2,50 & 7 & 0,70 \\
\hline 5 & & 103 & 10,00 & 70 & 6,80 & 25 & 2,40 & 53 & 5,10 & 37 & 3,60 & 14 & 1,40 \\
\hline 6 & & 96 & 9,30 & 43 & 4,20 & 18 & 1,70 & 37 & 3,60 & 46 & 4,40 & 20 & 1,90 \\
\hline 7 & & 96 & 9,30 & 32 & 3,10 & 10 & 1,00 & 36 & 3,50 & 45 & 4,30 & 20 & 1,90 \\
\hline 8 & & 77 & 7,40 & 14 & 1,40 & 9 & 0,90 & 41 & 4,00 & 51 & 4,90 & 31 & 3,00 \\
\hline 9 & & 43 & 4,20 & 13 & 1,30 & 7 & 0,70 & 35 & 3,40 & 62 & 6,00 & 37 & 3,60 \\
\hline 10 & & 43 & 4,20 & 12 & 1,20 & 4 & 0,40 & 27 & 2,60 & 65 & 6,30 & 51 & 4,90 \\
\hline 11 & & 20 & 1,90 & 5 & 0,50 & 4 & 0,40 & 22 & 2,10 & 80 & 7,70 & 48 & 4,60 \\
\hline 12 & & 19 & 1,80 & 4 & 0,40 & 6 & 0,60 & 18 & 1,70 & 65 & 6,30 & 57 & 5,50 \\
\hline 13 & & 7 & 0,70 & 1 & 0,10 & 1 & 0,10 & 11 & 1,10 & 79 & 7,60 & 63 & 6,10 \\
\hline 14 & & 7 & 0,70 & - & - & 1 & 0,10 & 12 & 1,20 & 66 & 6,40 & 69 & 6,70 \\
\hline 15 & & 1 & 0,10 & 1 & 0,10 & 1 & 0,10 & 1 & 0,10 & 64 & 6,20 & 78 & 7,50 \\
\hline 16 & & 3 & 0,30 & 3 & 0,30 & 1 & 0,10 & 5 & 0,50 & 59 & 5,70 & 73 & 7,10 \\
\hline 17 & & 1 & 0,10 & 1 & 0,10 & - & - & 2 & 0,20 & 43 & 4,20 & 73 & 7,10 \\
\hline 18 & & 1 & 0,10 & - & - & - & - & - & - & 54 & 5,20 & 63 & 6,10 \\
\hline 19 & & - & - & - & - & - & - & 1 & 0,10 & 36 & 3,50 & 62 & 6,00 \\
\hline 20 & & - & - & - & - & - & - & - & - & 30 & 2,90 & 53 & 5,10 \\
\hline 21 & & 1 & 0,10 & - & - & 1 & 0,10 & - & - & 19 & 0,80 & 44 & 4,30 \\
\hline 22 & & - & - & - & - & - & - & - & - & 21 & 2,10 & 44 & 4,30 \\
\hline 23 & & - & - & - & - & - & - & - & - & 13 & 1,30 & 38 & 3,70 \\
\hline 24 & & - & - & - & - & - & - & - & - & 8 & 0,80 & 27 & 2,60 \\
\hline 25 & & - & - & - & - & - & - & - & - & 5 & 0,50 & 22 & 2,10 \\
\hline 26 & & - & - & - & - & - & - & - & - & 5 & 0,50 & 13 & 1,30 \\
\hline 27 & & - & - & - & - & - & - & - & - & 3 & 0,30 & 7 & 0,70 \\
\hline 28 & : & - & - & - & - & - & - & - & - & 4 & 0,40 & 4 & 0,40 \\
\hline
\end{tabular}




\section{Tabela III}

Distribuição numérica e percentual dos dentes da arcada superior, por hemiarcada, segundo as condições em relação à cárie em 1035 jovens de 18 anos

- São Luís - Ma - 1978.

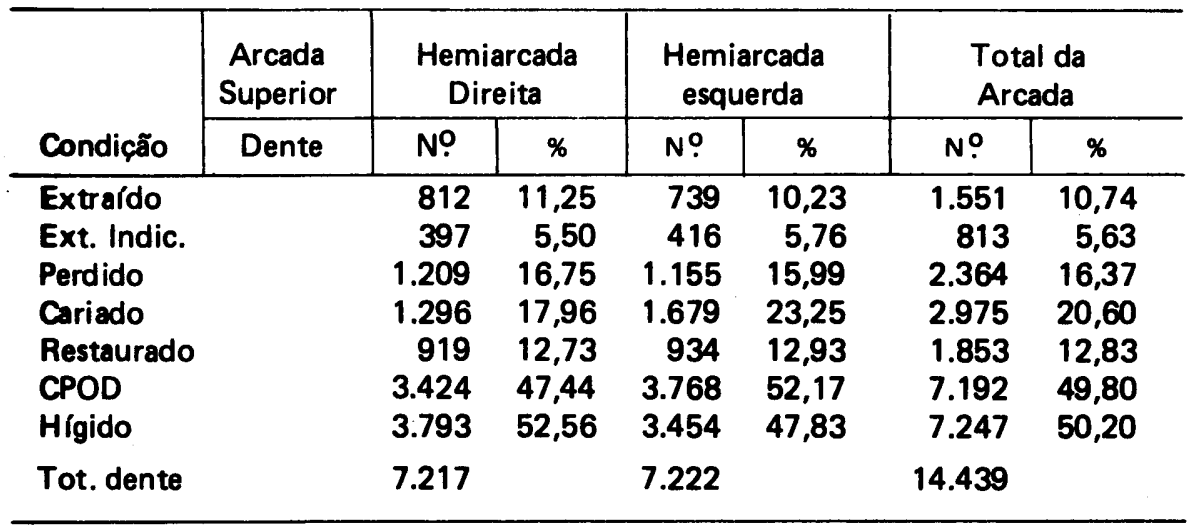

Tabela IV

Distribuição numérica e percentual dos dentes da arcada inferior, por hemiarcada, segundo as condições em relação à cárie em 1035 jovens de 18 anos

São Luís - Ma - 1978.

\begin{tabular}{|c|c|c|c|c|c|c|c|}
\hline \multirow[b]{2}{*}{ Condição } & \multirow{2}{*}{$\begin{array}{c}\text { Arcada } \\
\text { Inferior }\end{array}$} & \multicolumn{2}{|c|}{$\begin{array}{c}\text { Hemiarcada } \\
\text { Direita }\end{array}$} & \multicolumn{2}{|c|}{$\begin{array}{c}\text { Hemiarcada } \\
\text { Esquerda }\end{array}$} & \multicolumn{2}{|c|}{$\begin{array}{l}\text { Total da } \\
\text { Arcada }\end{array}$} \\
\hline & & No? & $\%$ & N? & $\%$ & N? & $\%$ \\
\hline $\begin{array}{l}\text { Extraido } \\
\text { Ext. Indic. } \\
\text { Perdido } \\
\text { Cariado } \\
\text { Restaurado } \\
\text { CPOD } \\
\text { Hígido }\end{array}$ & & $\begin{array}{r}943 \\
337 \\
1.280 \\
952 \\
598 \\
2.830 \\
4.408\end{array}$ & $\begin{array}{r}13,03 \\
4,66 \\
17,60 \\
13,15 \\
8,26 \\
39,10 \\
60,90\end{array}$ & $\begin{array}{r}885 \\
319 \\
1.204 \\
961 \\
626 \\
2.791 \\
4.444\end{array}$ & $\begin{array}{r}12,24 \\
4,41 \\
16,65 \\
13,28 \\
8,66 \\
38,58 \\
61,41\end{array}$ & $\begin{array}{r}1.828 \\
656 \\
2.484 \\
1.913 \\
1.224 \\
5.621 \\
8.852\end{array}$ & $\begin{array}{r}12,63 \\
4,55 \\
17,18 \\
13,21 \\
8,45 \\
32,84 \\
61,16\end{array}$ \\
\hline Tot. dente & & 7.238 & & 7.235 & & 14.473 & \\
\hline
\end{tabular}


Dentes Restaurados

Somente $45,50 \%$ das pessoas examinadas não possuíam dentes restaurados; $8,60 \%$ possuíam apenas um dente restaurado; $6,60 \%$ e $6,30 \%$ estavam com 2 e 3 dentes respectivamente nessa condição. Uma pessoa estava com 19 dentes restaurados (Tab. II).

Houve maior porcentagem de dentes restaurados na arcada superior $(12,83 \%)$, que na inferior $(8,45 \%)$.

A hemiarcada superior esquerda apresentou maior número de dentes restaurados (correspondente a $12,93 \%)$; ficando a inferior direita com a menor porcentagem $(8,26 \%$ Tab. III e IV).

\section{Dentes CPOD}

Dos 1035 examinados, apenas 5 não possuíam dentes com experiência de cárie, sendo de $99,50 \%$ o ataque da cárie nessa população (Tab. II).

Os maiores valores percentuais de pessoas examinadas concentraram-se nos grupos que possuíam entre 6 e 18 dentes afetados pela cárie. Esses valores oscilaram de $4,30 \%$ a $7,70 \%$ para cada grupo, sendo que o valor mais alto foi para o grupc de 11 dentes. Quatro pessoas possuíam todos os 28 dentes afetados pela cárie.

A arcada superior teve maior experiência de cárie já que sua hemiarcada esquerda apresentou $52,17 \%$ e sua direita $47,44 \%$ dos seus dentes nessa condição. Das hemiarcadas, a inferior esquerda teve menor número de dentes afetados por cárie $(38,58 \%$ - Tab. III e IV).

Nas hemiarcadas superiores, as condições estudadas ficaram na seguinte ordem decrescente: cariados, restaurados, extraídos e extração indicada.
Nas hemiarcadas inferiores, os maiores valores continuaram sendo dos cariados e os menores, os de extração indicada; sendo que o número dos extraídos superou ao dos restaurados.

\section{Dentes Hígidos}

Quatro pessoas não possuíam dentes hígidos. Três possuíam somente um dente hígido, cinco possuíam 2 e cinco possuiam 3 dentes nessa condição.

As maiores porcentagens de examinados por dente hígido, foram: $7,50 \% ; 7,10 \% ; 7,10 \%$ e $6,70 \%$ que possuiam 15, 16, 17, e 14 dentes, respectivamente (Tab. II).

Observa-se (Tab. IV) que 61,16\% dos dentes inferiores estavam hígidos, enquanto que apenas $50,20 \%$ dos dentes superiores não foram afetados pela cárie (Tab. III).

Para dentes hígidos, a maior porcentagem, na arcada superior, foi do lado direito $(52,56 \%)$; e na inferior, do lado esquerdo $(61,49 \%$ Tab. III e IV).

\section{Testes}

Com a finalidade de testar se a localização, na hemiarcada, de dentes perdidos, cariados, restaurados e hígidos, apresenta associação, optou-se por um teste de $\chi^{2}$ (quiquadrado), ao nivel de significância de $1 \%$, a partir dos resultados da tabela que se segue: 
Tabela V

Distribuição do número de dentes perdidos, cariados, restaurados e hígidos, por hemiarcada, de 1035 jovens de 18 anos

S. Luis - Ma. - 1978

\begin{tabular}{l|c|c|c|c}
\hline \multirow{2}{*}{ Condição } & \multicolumn{2}{|c|}{ Arcada Superior } & \multicolumn{2}{c}{ Arcada Inferior } \\
\cline { 2 - 5 } & direita & esquerda & direita & esquerda \\
\hline Perdido & 1.209 & 1.155 & 1.280 & 1.204 \\
Cariado & 1.296 & 1.679 & 952 & 961 \\
Restaurado & 919 & 934 & 598 & 626 \\
Hígido & 3.793 & 3.454 & 4.408 & 4.444 \\
\hline
\end{tabular}

O valor calculado de $\chi^{2}=573,98$, foi superior ao $\chi^{2}=21,67$, para 9 graus de liberdade. Por isso, podemos admitir as conclusões do presente trabalho, para os dados da população estudada ao nível de significância de 0,01 .

Quadro comparativo do índice CPOD e sua composiç̃o percentual, de alguns trabalhos e da própria pesquisa. Porto Alegre 1978.

\begin{tabular}{|c|c|c|c|c|c|c|c|}
\hline \multirow{2}{*}{ Autor } & \multirow{2}{*}{ CPOD } & \multicolumn{2}{|c|}{ Cariado } & \multicolumn{2}{|c|}{ Perdido } & \multicolumn{2}{|c|}{ Roswerado } \\
\hline & & Valor & $\%$ & Valor & $\%$ & Valor & $\%$ \\
\hline $\begin{array}{l}\text { Campanella, S. \& Ciciarelli, A.M. (4) } \\
\text { Chaves, P.O. (7) } \\
\text { Hansen, B.F. (9) } \\
\text { Johnson, E.S. et alii (12) } \\
\text { Medeiros, E.P.G. (13) } \\
\text { Mejia, V.R. et alii (14) } \\
\text { Pinto, V.G. (16) } \\
\text { Câmara Filho, P. (autor da pesquisa) }\end{array}$ & $\begin{array}{l}11,83 \\
12,12 \\
25,70 \\
20,40 \\
15,04 \\
15,40 \\
14,81 \\
12,37\end{array}$ & $\begin{array}{l}5,51 \\
7,20 \\
5,10 \\
1,20 \\
6,37 \\
4,00 \\
3,38 \\
4,72\end{array}$ & $\begin{array}{r}46,56 \\
59,41 \\
19,84 \\
6,00 \\
42,35 \\
25,97 \\
22,82 \\
38,16\end{array}$ & $\begin{array}{r}1,31 \\
3,70 \\
1,70 \\
13,50 \\
7,17 \\
10,40 \\
10,00 \\
4,68\end{array}$ & $\begin{array}{r}11,07 \\
30,53 \\
6,62 \\
66,18 \\
47,67 \\
67,53 \\
67,48 \\
37,83\end{array}$ & $\begin{array}{r}5,01 \\
1,22 \\
18,90 \\
5,70 \\
1,50 \\
1,00 \\
1,43 \\
2,97\end{array}$ & $\begin{array}{r}42,36 \\
10,07 \\
73,54 \\
27,94 \\
8,97 \\
6,49 \\
9,66 \\
24,01\end{array}$ \\
\hline
\end{tabular}

\section{6 - DISCUSSÃO}

Não parece válido comparar cárie em idades diferentes, mesmo que todas as outras variáveis sejam iguais, porque, sendo ela um fator que interfere no resultado - variável depen. dente - impossibilitará ou mascarará a comparação.

Contudo, não havendo abundan. tes estudos de cárie em idade de 18 anos, foram obtidos dados também de idades anteriores e de faixas etárias que a englobam.

$\mathrm{Na}$ comparacão desses dados com os obtidos neste trabalho, pode ser observada a existência de coerência dos resultados desta pesquisa:

Enquanto em Bogotá (14), em 1971, havia $96,19 \%$ das pessoas de 15 a 19 anos atingidas pela cárie, na população aqui estudada, $99,50 \%$ o 
foram. Pouco faltou para que esta população estivesse com $100,00 \%$ das pessoas afetadas por cárie, enquanto, em Bogotá, essa totalidade só foi alcançada aos 45 anos de idade. Portanto, a população em estudo alcançou nivel de ataque de cárie, com apenas $0,50 \%$ de diferença da observada em população colombiana de 27 anos mais velha.

Para a ação da cárie em relação ao total dos dentes o resultado encontrado $(44,31 \%)$ foi próximo do encontrado por CAHEN et alii (3), para 18 anos $(43,50 \%)$; maior que o encontrado por JACKSON (11), para faixa etária de 15 a 19 anos $(37,00 \%)$ e dos 39,41\% encontrados por CAMPANELLA \& CICIARELLI (4), para a idade de 14 anos; mas foi menor do que os 49,00\% na Colōmbia, encontrado por MEJIA et alii (14), para o grupo de 15 a 19 anos. Portanto, a população aqui estudada, está menos afetada por cárie que as estudadas por CAMPANELLA \& CICIARELLI (4) e por MEJIA (14); e mais ou menos igual às demais.

\section{CPOD}

$O$ indice CPOD 12,37 é bastante coerente com os encontrados nạ literatura. $O$ único estudo que apresenta dados da mesma idade (18 anos) foi o de CAHEN et alii (3), cujo resultado $(12,60)$ difere apenas de 0,23 , deste.

SUMMERS (19) estudou população de 12 anos e encontrou valores CPOD variando de 3,60 a 5,89 . Fez estudos além de ser em população que utilizava água fluoretada artificialmente, utilizando crianças de cor branca e de cor preta, que geralmente têm menor número de cárie que crianças brancas, segundo comprovou e foi confirmado por SOUSA et alii (18), MOREIRA \& VIEIRA (15) e BAGRAMIAN \& RUSSEL (1).

SOUSA'et alii (18) também estudaram populações de 12 anos, divididas em brancas e não brancas, obtendo valores compreendidos entre 6,76 e 8,58 para o CPOD.

MOREIRA \& VIEIRA (15) estudando cárie em escolares de 12 anos de cor branca e de cor preta obtiveram valores para CPOD variando de 6,83 a 9,43 , para ambos os sexos.

Vê-se que, para uma idade que corresponde a $2 / 3$ da que foi estudada neste trabalho, os valores encontrados por SOUSA et alii (18) e por MOREIRA \& VIEIRA (15) são bem próximos e variaram de aproximadamente, $2 / 3$ a $3 / 4$ do CPOD desta pesquisa $(12,37)$.

A diferença entre os valores de CPOD do estudo de SUMMERS (19) e o deste trabalho pode ser atribuído ao fato de sua população ser beneficiada pelo fluor da água utilizada.

Já CAMPANELLA \& CICIARELLI (4) encontraram CPOD igual a 11,83, para a idade de 14 anos. Idade mais próxima de 18 anos e o indice mais próximo do aqui encontrado.

PINTO (16) encontrou, para população de 15 anos, em Passo das Pedras - Porto Alegre - RS (Br), CPOD de 14,81 , portanto, maior que o da população aqui estudada. Este resultado leva-nos a esperar que, naquela Vila, a população de 18 anos tenha um índice de cárie bem maior que o da população aqui em estudo.

BAGRAMIAN \& RUSSEL (1) estudaram população de 17 anos e obtiveram indices CPOD com valores 
(de 10,22 a 12,83), próximos do encontrado nesta pesquisa. Mesmo tendo sido de duas cidades diferentes, para raças diferentes, vê-se a coerência dos valores daqueies com o desta pesquisa.

Os autores que estudaram a idade de 18 anos, incluída em faixas etárias, obtiveram valores com os quais os deste trabalho são coerentes, por exemplo:

CHAVES (7) englobou os examinados em grupos de idades e, para o de idades de 16 a 21 , obteve CPOD igual a 12,12, valor, que se aproxima muito do aqui encontrado, visto que estão incluídas 3 idades antes e 3 idades depois de 18 anos, o que poderia dar a média para essa idade.

MEDEIROS (13) encontrou um CPOD de 15,04 para a população de 18 a 20 anos; o que é viável, comparado com o desta pesquisa $(12,37)$, visto ter ele acrescido mais duas idades além dos 18 anos.

JACKSON (11) afirma que o índice CPOD cresce com a idade, o que é confirmado por GUIMARÃES (8) em seu estudo de risco de cárie. Por isso, o valor encontrado por MEDEI. ROS (13) deveria ser, realmente um pouco maior que o deste trabalho. Outro exemplo é o de HANSEN (9), que, examinando população de 35 anos, encontrou CPOD com valor de 25,70 .

No estudo de MEJIA et alii (14), que alcançou idades de 5 a mais anos, o índice CPOD obtido foi de 15,40. Em termos gerais, é viável esse valor, visto que apesar de estarem incluídas as pessoas menores de 18 anos de idade, que possuem índice menor, também foram incluídas pessoas de idade maior que o desta idade.
O estudo em adultos nos Estados Unidos da América do Norte, feito por JOHNSON et alii (12), confirma a coerência do valor de 12,37 para CPOD de 18 anos, pois revelou um valor maior para o CPOD $(20,40)$, plenamente justificável, visto que a idade da população foi de 18 a 79 anos. Dessa mesma pesquisa, dados publicados em 1967, pelo Serviço de Saúde Pública dos Estados Unidos da América do Norte (21) revelaram que, embora em homens e muIheres jovens (sem precisar a idade) tivessem sido encontrados índices CPODs de 13,40 e 14,10, na faixa etária de 75 a 79 anos, esse índice chegou a 25,00 .

\section{Dentes perdidos}

No $\operatorname{CPOD}(25,70)$ encontrado por HANSEN (9), para 35 anos, havia apenas $6,62 \%$ de perdidos.

Por outro lado, em Passo das Pedras (16), em população de 15 anos, $67,48 \%$ do CPOD foi de perdidos, expressando grande perda de dentes. $O$ mesmo aconteceu com a população de Bogotá (14) onde, para a faixa etária de 15 a 19 anos, $67,53 \%$ do CPOD $(15,40)$ eram de perdidos (Quadro Comparativo).

Porcentagem próxima desta última $(66,18 \%)$, foi obtida nos Estados Unidos (12) , para a faixa etária de 18 a 79 anos.

Neste trabalho, $37,83 \%$ do CPOD $(12,37)$ encontrado era de perdidos. Esse percentual foi menor do que o de perdidos $(47,67 \%)$ no $C P O D$ encontrado por MEDEIROS (13) para a faixa etária de 18 a 20 anos. Mas foi maior que os $30,53 \%$ de perdidos do CPOD encontrado por CHAVES (7), para as idades de 16 a 21 anos: bem maior ainda que os $11,07 \%$ de 
perdidos, observados por CAMPANELLA \& CICIARELLI (4), no índice de cárie de pessoas de 14 anos (Quadro Comparativo).

\section{Cariados}

Mais da metade dos dentes CPOD da população estudada por CHAVES (7) era de cariados $(59,41 \%)$. Também (com $46,56 \%$ de cariados no CPODI, a população estudada por CAMPANELLA \& CICIARELLI (4) estava mais carente de restaurações dentárias, que a estudada neste trabaIho. (Quadro Comparativo).

A população do presente estudo estava com $38,16 \%$ dos dentes CPOD constituído de cariados; valor pouco abaixo de $(42,35)$ encontrado por MEDEIROS (13) para o correspondente CPOD. (Quadro Comparativo).

A população que menos possuía dentes com cárie foi a estudada por JOHNSON et alii (12), com apenas $6,00 \%$ do CPOD. Ressalve-se que $66,18 \%$ desse indice era de perdidos.

Valores intermediários estavam nas populações estudadas por HANSEN (9); por PINTO (16) e por MEJIA (14), respectivamente, com $19,84 \% ; 22,82 \%$ e $25,97 \%$ de cariados nos índices de ataque de cárie (Quadro Comparativo).

\section{Restaurados}

Das referênçias citadas neste trabalho, apenas a de HANSEN (9) apresentou população com um grande número de dentes restaurados (73,54\% do CPOD), com 18,9 dehtes, em média por pessoa.

A 2. maior porcentagem de restaurados $(42,36 \%$ do índice CPOD) foi a da população estudada por CAMPANELLA \& CICIARELLI (4), com um grau médio de atendimento restaurador. Cumparando-se os dois estudos anteriores e o de JOHNSON et alii (12), onde apenas $27,94 \%$ do CPOD $(20,40)$ eram de restaurados, vê-se que a estudada neste trabalho está em situação pior que aquelas em termos de odontologia restauradora, porque, apenas $24,01 \%$ do CPOD eram de dentes com restauração.

As populações estudadas por CHAVES (7), por MEDEIROS (13) e por PINTO (16) apresentaram pequeno grau de atenção de odontologia restauradora, pois apenas $10,07 \%$; $9,97 \%$ e $9,66 \%$ dos respectivos CPODs eram de dentes restaurados.

A população menos beneficiada por trabalhos de recuperação dental foi a de MEJIA et alii (14), onde apenas $6,49 \%$ dos dentes afetados por cárie estavam restaurados (Quadro Comparativo).

Comparando os resultados deste estudo com os dos estudos aqui citados, que foram feitos em população brasileira, em termos de necessidade de serviço $(C+E i)$, pode ser observado que :

- na estudada por MEDEIROS (13) $50,40 \%$ do CPOD $(15,04)$ necessitava de extração e de restaurações $(C+E i=7,58)$; praticamente igual à estudada por CAMPANELLA \& CICIARELLI (4), onde $50,38 \%$ do índice CPOD $(11,83)$ estava nessa condição $(C+E i=5,96)$ e bem próxima da estudada aqui, na qual $49,64 \%$ do CPOD $(12,37)$ também necessitavam daqueles cuidados $(\mathrm{C}+$ $\mathrm{Ei}=6,14)$.

O estudo feito por PINTO (16) apresentou apenas $33,56 \%$ do CPOD $(14,81)$ com necessidades de extração e de restaurações $(C+E i=4,97)$, o que poderia dar idéia de uma população melhor assistida odontologica- 
mente. Entretanto, quando se observa que $56,79 \%$ do índice de cárie $(E=8,41$ em um $C P O D=14,81)$ era de dentes extraídos, conclui-se que essa população recebeu quase que exclusivamente odontologia radical, o que se constitui na retirada de um problema odontológico - a dor de dente - e na introdução de outros, como o fonético, o estético, o fisiológico e o psicológico.

\section{7 - CONCLUSÕES}

1 - 0 ataque de cárie na população estudada chegou a $99,50 \%$, dando a média de 12,37 dentes por pessoa ;

$2-44,31 \%$ dos 28.912 dentes erupcionados, na amostra em estudo, já haviam sido atacados pela cárie ;

3-o CPOD foi, estatisticamente, maior, ao nível de significância de $1 \%$, na arcada superior e, nesta, do lado esquerdo; enquanto na arcada inferior, o lado direito teve maior ocorrência;

4 - a perda de dentes foi maior, estatisticamente, ao nível de $1 \%$, em dentes inferiores $(17,18 \%)$ que em superiores $(16,37 \%)$ e, em ambas as arcadas, o lado direito teve mais perdidos:

5 - cada pessoa teve classificados como perdidos, em média, 4,68 dentes;

6 - em média, cada pessoa havia extraído 3,26 dentes, e a arcada inferior apresentou valores estatisticamente maiores, ao nível de $1 \%$;

7 - houve mais extraídos do lado direito em ambas as arcadas, estatisticamente, ao nível de $1 \%$ de significância;

$8-15,70 \%$ dos examinados não tinham nenhum dente extraído:

9 - ao nível de significância de
$1 \%$, houve mais extração indicada na arcada superior e, nesta, no lado esquerdo;

$10-50,10 \%$ dos examinados necessitava de extração e, em média, cada um, de 1,42 dentes;

$11-4,72$ dentes por pessoa estavam com cárie e a arcada superior teve 143 a mais que a inferior;

12 - em ambas as arcadas, o lado esquerdo apresentou, estatisticamente, mais dentes cariados, ao nível de significância de $1 \%$, sendo que, na superior, a diferença entre as hemiarcadas foi maior;

$13-2,97$ dentes, em média por pessoa, estavam restaurados, mas $45,50 \%$ dos examinados não possuíam nenhum dente com restauração;

14 - a arcada superior teve $1 / 3$ a mais dentes restaurados, e as hemiarcadas esquerdas tiveram valores pouco maiores que as direitas; $\mathrm{e}$

15 - cada examinado possuía, em média, 15,55 dentes hígidos, havendo maior ocorrência na arcada inferior, e, nesta, na hemiarcada esquerda.

\section{SUMMARY}

1035 students in third grade of secondary school, in São Luís, the capital of Maranhão, Brazil, were examined. The mean age of them was about 18 years old. Utilizing a plan mirror and explorers number 5 , each student in the study received a clinical dentel examination for diagnosing caries prevalence, through the DMF index and its components, by half maxilla and half jaw. In 99,50 per cent of the population studied, an average of caries incidence was 12,37 DMF by person and dental caries reached 44,31 per cent of erup- 
ted teeth. The highest caries inciden$c e$ was on the superior arcade, in special in the left half maxilla. On the average, each student had 4,68 lost teeth; 4,72 with caries; and 2,87 restered. 15,10 per cent did not hawe removed teeth and 50,10 per cent needed at least, dental extraction. 45,50 per cent did not have restoration.

\section{REFERENCIAS BIBLIOGRAFICAS}

1 - BAGRAMIAN, R.A. \& RUSSELL, A.L.. An Epidemiologic study of dental corie in race and geografic area. J. Dent. Res. 50(6):1553-6. Nov./Dec. 1971.

2 - BUNERR, A.V. \& SCHEEP, H.W. Oral microbiology infecton diseases. 7.ed. Baltimore, Williams \& Wilkins, 1963. 1003p.

3 - CAHEN, P.M.; CAUBERT, A.M.; and FRANK, R.M. Oral condition in a population of young adults in Strasbourg. Community Dent. and Oral Ep. Copenhagem, 5(1):40-5, Jan. 1977.

4 - CAMPANELLA, S. \& CICIARELLI, J.R.R. Análise dos dados de prevalência de cárie dental na Cidade de Guaira, SP. Brasil, em 1710 escolares de ambos os sexos, segundo a idade. Rev. Ass. Paul. Cir. Dent., 31(5) :313-21. set./out. 1977.

5 - CHAVES, M.M. Odontologia sanitária. Washington. Organizacion Panamericana de la Salud. Oficina Sanitária Panamericana. Oficina Regional de la OMS., jul. 1962.

6 - _ Manual de odontologia sanitária. S.Paulo, Faculdade de Higiene e Saúde Pública da Universidade de São Paulo, 1960. p.45.

7 - CHAVES, P.O. Contribuição à educą̧ão odontológica. Experiência de um programa incremental. Porto Alegre, 1963. Tese de Livre-Docência para a Faculdade de Odontologia da UFRGS.

8 - GUIMARÃES, L.O.C. Risco de ataque da cárie em dentes permanentes. Rev. Fac. Odont. S.Paulo, 1012) 201-20, jul./dez. 1972.

9 - HANSEN, B.F. Caries experience in a Norwegian urban population. Community Dent. Oral Epidemiol, 5(3):132-5, May 1977.

10 - HILL, I.N.; BLAYNEY, J.R.; WOLF, W. Evanston dental caries study. XVII Dental caries experiences rates associated with boys and girls, white and black children. J. Am. Dent. Assoc., 55:473-82. Oct. 1957.

11 - JACKSON, D. An Epidemiological study of dental caries prevalence in adults. Arch. Oral Biol. 680-93, 1961, Special Suppl.

12 - JOHNSON, E.S. et alii. Selected dental findings for adults. In: U.S.A. Department of Health Education, and Welfare. Public Health Service. Selected dental findings in adults by age, race and sex; United States 1960-1962. Washington, 1965. 35p. - (National Center for Health Statistics. Set. 11(7)).

13 - MEDEIROS, E.P.G. Necessidades de tratamento odontológico em operários de São Paulo. Rev. Bras. de Odont. 171:13-21. Såo Paulo. set./out. 1971. 
14 - MEJIA, V.R. et alii. - Caries dental. In: COLOMBIA. Ministério de Salud Pública \& Associacion de Faculdad de Medicina. Estudio de recursos humanos para la salud y educacion medica em Colombia. Bogotá, 1971. p.7-9 e 29-32.

15 - MOREIRA, B.H.W. \& VIEIRA, S. Prevalence of dental caries in permanent teeth of white and black school children in Brazil. Community. Dent. Oral. Epidemiol., Copenhagem: 5(3):129-31. 1977.

16 - PINTO, V.G. Assistência odontológica em núcleos populacionais. In: PORTO ALEG RE. Prefeitura Municipal. Secretaria Municipal de Saúde e Serviço Social. Divisão de Saúde e Serviço Social. 20. Encontro de Saúde Pública em Odontologia. Porto Alegre. 1971. p.22-31.

17 - RIESER, J. Observações sobre a não incidência de cárie em certas regiőes do Nordeste do Brasil. Seleçбes Odontologicas. 6(31):49-74, jul./ago. 1951.

18 - SOUSA, J.M.P.; ARRILLAGA, N.N.; UCHÓ, F.V.; ROCHA, O. Prevalência de cárie dental em brancos e não brancos. Rev. Saúde Púb. 1(1):38-43, jun. 1967.

19 - SUMMERS, G.J. Dental needs and prevalence of dental caries in Wayne Country Grade School Children. J. Mich. Dent. Assoc., 52(5):127-35. May 1970.

20 - TINOCO, A.F. et alii. Baixa prevalência de cárie dentária na Cidade de Areia Branca. Estado do Rio Grande do Norte. Rev. da Assoc. Paul. de Cir. Dent, 20(4):170-3, 1966.

21 - U.S.A. Department of Health, Education and Welfare Vital and Health Statistics. Decayed, missing and filled teeth in adults. United States 1960-1962. Washingtan, 1967. (Feb. Public. Health Service National Center of Health Statistics. Series 11(23)).

22 - WAYGH, L.M. A Study of nutrition and teeth of Eskimo of North Bering sea and Artic Alaska. J. of Dent. Res. 10:387-92. 1930. 